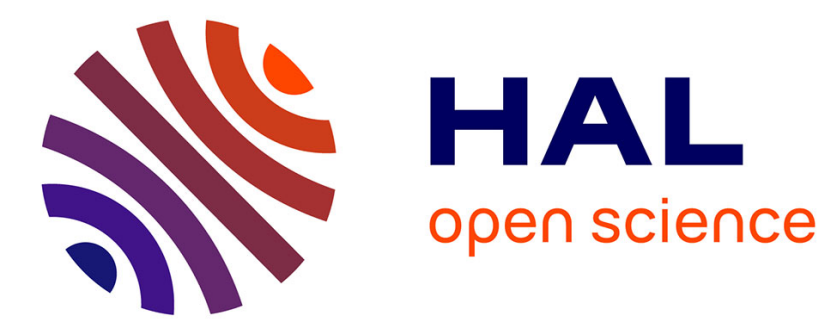

\title{
Sur le front des frontières
}

Emmanuel Blanchard, Olivier Clochard, C. Rodier

\section{To cite this version:}

Emmanuel Blanchard, Olivier Clochard, C. Rodier. Sur le front des frontières. Plein Droit, 2010, 87, http://www.gisti.org/spip.php?article2119. 10.3917/pld.087.0003 . hal-00746526

\section{HAL Id: hal-00746526 https://hal.science/hal-00746526}

Submitted on 29 Oct 2012

HAL is a multi-disciplinary open access archive for the deposit and dissemination of scientific research documents, whether they are published or not. The documents may come from teaching and research institutions in France or abroad, or from public or private research centers.
L'archive ouverte pluridisciplinaire HAL, est destinée au dépôt et à la diffusion de documents scientifiques de niveau recherche, publiés ou non, émanant des établissements d'enseignement et de recherche français ou étrangers, des laboratoires publics ou privés. 
Dernier ajout : mercredi 15 décembre 2010, 17:42

URL de cette page : http://www.gisti.org/spip.php?article2119

Accueil du site $>$ Publications $>$ Plein Droit $>$ Numéros $>87$

Plein droit, 87, décembre 2010

«Sur le front des frontières»

\title{
Sur le front des frontières
}

\author{
Dossier coordonné par Emmanuel Blanchard, Olivier Clochard et Claire Rodier \\ Membres de Migreurop
}

\begin{abstract}
Avec le développement des migrations et, surtout, de leur contrôle, la définition de la frontière, longtemps centrée sur des critères géographiques et techniques, a évolué, intégrant de nombreux autres facteurs (politiques, économiques, juridiques...). Des dispositifs anti-immigration ont alors fait leur apparition dans de nombreux endroits du monde, dans le but de juguler les flux d'u indésirables ". Barrières classiques (murs, grillages, barbelés), " biométrisation ", multiplication des mécanismes de contrôle à distance, les frontières se matérialisent aujourd'hui par toute une série d’obstacles dans les parcours des exilés à la recherche d'une vie meilleure ou d'une protection.
\end{abstract}

Dans certains grands aéroports internationaux, les contrôles de police ont disparu pour une partie des voyageurs qui traversent les frontières en quelques instants. Ainsi à Roissy-Charles-de-Gaulle, le système Parafes (passage automatisé rapide aux frontières extérieures Schengen) permet aux ressortissants de l'Union européenne volontaires une « inscription » préalable est nécessaire - de se contenter de traverser un portique dans lequel ils font reconnaître la bande optique de leur passeport et les empreintes de huit de leurs doigts. L'authentification biométrique est censée effacer les frontières et ouvrir un vaste espace mondialisé pour celles et ceux qui acceptent de se soumettre à ces dispositifs. La conjonction de l'anthropométrie née à la fin du xixe siècle [1] et de la puissance de calcul de l'informatique du xxie siècle - les bases de données sont les nouvelles barrières à la mobilité - est cependant loin de favoriser la liberté de circulation : des exilés du monde entier cherchent, en s'automutilant, à effacer leurs traces afin d'échapper à la surveillance du fichier Eurodac. Destiné à stocker les empreintes de tous les demandeurs d'asile et des migrants « irréguliers » interpellés lors des contrôles, ce fichier est une véritable épée de Damoclès au-dessus de celles et ceux qui ont eu le malheur d'entrer par les mauvaises portes de l'Europe, par les interstices des îles grecques ou d'autres espaces investis par les damnés de la mondialisation. Dans leur cas, l'identification biométrique débouche sur les « reconduites à la frontière » et autres modes d'assignation à résidence des exilés.

Aujourd'hui comme hier, la technologie des frontières est ségrégationniste : les postes-frontières français qui, au début du xxe siècle, servaient à retenir les caravanes de « romanichels » [2] ont certes été démantelés, mais les itinérants et les minorités nationales, dont les «Roms » sont emblématiques, ne bénéficient toujours pas de l'effacement progressif des délimitations nationales à l'intérieur de l'espace Schengen. II ne faudrait d'ailleurs pas s'arrêter aux apparences de la modernité politique et technologique du gouvernement des frontières : les vieilles séparations entre États sont loin d'avoir disparu et se sont même démultipliées. Depuis 1991 et, notamment, l'éclatement de l'URSS, de la Yougoslavie et de la Tchécoslovaquie, $26000 \mathrm{~km}$ de frontières ont été instituées dans le monde et 24000 ont fait l'objet d'accords de délimitation [3]. Surtout, leur surveillance continue en maints endroits d'obéir à un archaïsme de la force pure qui assimile les « indésirables » de la mobilité internationale des « vies sans valeur ». La pluralité des frontières est d'ailleurs au coeur des formes contemporaines de gouvernement des populations. Ainsi l'Inde qui mure, grillage de barbelés et sature d'hommes en armes les points de passage avec le Bangladesh et le Pakistan, s'est aussi lancée dans le recensement biométrique de l'ensemble de sa population (dont les déplacements internes ont toujours été vus comme un ferment de déstabilisation sociale) et la « traçabilité » des mouvements de tous les étrangers pénétrant sur son territoire [4]. Les anciennes délimitations géographiques se déplient aujourd'hui pour pister les mouvements de populations et non plus simplement les arrêter.

\section{Les frontières des géographes}

La définition des frontières a longtemps été guidée par des facteurs exclusivement géographiques et techniques. Au cours de la seconde moitié du xxe siècle, des géographes [5] vont en proposer une idée beaucoup plus riche en faisant référence à un ensemble d'éléments politiques, économiques, sociologiques, juridiques, etc. Pour Paul Guichonnet et Claude Raffestin [6], « toutes les définitions [de la frontière] ou presque s'appuient sur la notion de zone, de mouvement et de souveraineté. Autrement dit, il y a référence à l'espace, à la "cinématique" et au droit ». Dans cette perspective et du fait de la multiplication des contrôles des flux migratoires depuis les années 1990, on note qu'en de 
nombreux endroits du monde, des dispositifs « anti-immigration » tentent de juguler des flux d'« indésirables ». Dans le même temps, d'autres réalités frontalières réapparaissent de part et d'autre de ces limites internationales, sous diverses formes, "mais toujours en des lieux où les contrôles migratoires peuvent s'opérer et être efficaces. Ces réalités de la frontière, qui ne sont pas exclusives l'une de l'autre, ont tendance même à se combiner » [7]. Où se situent réellement les frontières qui font obstacle aux projets des personnes qui aspirent à une meilleure vie ailleurs ?

\section{Murs et zones tampons}

La première image d'une frontière est souvent celle d'une barrière et de guérites (les postes frontières), voire celle d'un mur ou d'une clôture grillagée surmontée de fils barbelés et ponctuée de check points. Cette représentation linéaire renvoyant notamment au rideau de fer qui a séparé l'Europe pendant près de trente ans - n'a pas disparu. Vingt ans après la chute de celui qui séparait l'Allemagne en deux, de nouveaux murs ont été érigés sur certaines lisières internationales, comme autour des possessions espagnoles en territoire marocain, les enclaves de Ceuta et Melilla, ou entre le Mexique et les États-Unis. Mais l'édification de ce type de barrières n'est pas l'apanage des " pays du Nord ». En 2003, le Botswana a installé un grillage d'une longueur de près de 500 kilomètres sur sa frontière avec le Zimbabwe, officiellement pour contenir l'épidémie de fièvre aphteuse, officieusement pour limiter l'afflux des Zimbabwéens fuyant la crise économique [8]. L'Inde a aussi dressé en 2007 une barrière de $4000 \mathrm{~km}$ afin d'empêcher les migrants bangladais d'atteindre son territoire [9].

Aux abords de ces éloquentes lignes de fractures apparaissent des zones tampons, qu'accompagne souvent l'installation de campements informels comme au nord du Maroc ou dans la partie septentrionale du Mexique. Ces murs participent à des systèmes qui généralisent la mise à l'écart des plus pauvres, avec d'un côté des sanctuaires de pays « riches ", et de l'autre des zones d'où il sera interdit de sortir, au mépris du droit de chacun " de sortir de tout pays, y compris le sien » [10]. Le contournement du droit international et de la souveraineté des États du Sud est d'ailleurs au coeur du processus de redéfinition des frontières par les dispositifs de contrôle migratoire.

\section{Les frontières externalisées}

Ces « discontinuités géographiques » sont par ailleurs soutenues en amont par d'autres mécanismes visant à lutter contre « l'immigration clandestine ». Les agents consulaires établissent les premiers contrôles à distance, tout comme les fonctionnaires des services d'immigration détachés dans les aéroports étrangers où sont susceptibles d'embarquer irrégulièrement des migrants à destination de leur pays - ou d'un État membre de l'Union européenne, s'agissant du corps européen d'officiers de liaison immigration mis en place depuis 2003. C'est aussi le cas des employés des agences de transport, contraints de vérifier que l'étranger qui souhaite voyager est en possession des documents de voyage requis pour l'entrée dans le pays de destination. À défaut, le transporteur qui l'aura acheminé non seulement devra le reprendre en charge sans délai et le ramener dans le pays de départ, mais risque en outre une amende très élevée. Cette privatisation des contrôles a été vivement critiquée par le commissaire aux Droits de l'homme du Conseil de l'Europe, qui estime que " la responsabilité de déterminer si tel ou tel migrant devrait avoir ou non la possibilité d'entrer sur un territoire ne doit pas reposer sur les épaules d'une compagnie de transport publique ou privée qui, à l'évidence, n'a pas vocation à le faire et n'en a pas les moyens » [11]. Depuis le 11 septembre 2001, la délocalisation de la surveillance des États « développés » dans des pays qui le sont moins s'est considérablement renforcée. La pluralité d'acteurs en postes avancés participe d'un phénomène de démultiplication et d'invisibilisation de la frontière au gré du déploiement des contrôles. Par exemple, dans de nombreux consulats à travers le monde, la délivrance des visas constitue aujourd'hui un outil essentiel de la politique migratoire des États membres de l'Union européenne (UE) [12]. Dans le cadre du partenariat qu'elle met en place avec ses voisins (politique de voisinage), l'UE exporte aussi une partie de ses contrôles dans des pays limitrophes comme le Maroc ou l'Ukraine. Le « rayon des contrôles migratoires " s'est donc considérablement élargi, les frontières étant devenues des instruments des politiques d'immigration et d'asile des États européens qui essaient d'en modifier la localisation et/ou les fonctions au gré de leurs besoins [13]. De la même façon, les États-Unis avec le Mexique, l'Australie avec la Papouasie-Nouvelle- Guinée ou Israël avec l'Égypte essaient-ils d'imposer leurs propres politiques sécuritaires et anti-migratoires à leurs voisins. Ainsi les contrôles s'effectuent-ils en de multiples lieux où le contrôle et l'expulsion des migrants sont réalisables, plus encore sur les frontières maritimes qui se matérialisent beaucoup plus difficilement que les frontières terrestres.

Selon la Convention des Nations unies sur le droit de la mer (CNUDM de 1982), l'État possède une zone contiguë pouvant aller jusqu'à 24 miles des côtes - dans laquelle il a le droit de réprimer les infractions à sa législation sur l'immigration. Or dans bien des cas, des opérations de surveillance en haute mer - parfois menées au nom de la lutte contre le terrorisme - font que les contrôles migratoires s'opèrent au-delà des 24 milles, parfois dans le cadre d'accords conclus avec les pays voisins (États-Unis avec Haïti, Italie avec Libye). Ces coopérations transfrontalières tracent de nouveaux périmètres qui se dissocient des parties continentales des États. 


\section{La criminalisation du franchissement des frontières}

Dans un processus de criminalisation de l'immigration, le franchissement des limites nationales par les étrangers dépourvus des documents exigés est, de plus en plus, associé à un fait délictueux [14]. Les lieux d'enfermement quelle que soit leur position géographique - sont par conséquent devenus, depuis une trentaine d'années, des éléments de la frontière, les États ayant construit des justifications légales à la détention des migrants. Les occupants de ces « camps migratoires » [15] sont confrontés à des mesures d'exclusion et d'interdiction. Officiellement, les conventions internationales qui protègent les droits humains ratifiées par les États ne sont pas remises en cause, mais une multitude de règles (légales ou non) élaborées depuis le milieu des années 1980, mettent à néant « le droit d'avoir des droits » selon l'expression d'Hannah Arendt. Au prétexte de lutter contre la fraude ou les abus, la possibilité de faire examiner sa demande d'asile quand on franchit la frontière d'un pays signataire de la convention de Genève de 1951 sur les réfugiés, et/ou de rentrer sur le territoire au titre du droit au rapprochement familial est refusée ou différée. La frontière peut être alors considérée comme lieu de condensation et de tension migratoire d'une part (comme dans les camps d'étrangers) et comme limite géographique à l'exercice du droit d'autre part. Ces " sas d'entrée », qui s'inscrivent dans les processus de délinéarisation des limites étatiques, deviennent des zones habitées et des étapes importantes dans le parcours migratoire.

Les usages administratifs issus des politiques d'asile et d'immigration concourent aussi à l'établissement de pratiques qui peuvent être qualifiées de frontalières, les dispositifs existant au sein même d'un territoire national (par exemple l'enregistrement d'une demande d'asile dans une préfecture) pouvant rappeler ceux mis en place aux frontières. Comme le traitement d'une demande d'un visa dans un consulat, ils constituent parfois des extensions de ce qu'on trouve aux limites des États. La force de ces contrôles connectés à des réseaux informatiques [16] tient aussi dans leur capacité à produire des effets dans plusieurs pays à la fois. Ils s'orientent ainsi vers une disposition de plus en plus réticulaire, mettant en oeuvre un ensemble de dispositifs interconnectés et réunis par les échanges d'informations sur les personnes que les États souhaitent expulser ou ne pas voir arriver sur leur territoire. Ainsi est envisagée, dans le cadre de la " gestion intégrée des frontières » de l'UE, la mise en place d'un registre électronique des entrées et sorties. Ce dernier s'appuie sur des éléments biométriques permettant l'enregistrement dans une base de données commune des informations relatives à la date et au lieu d'entrée, et à la durée de séjour de tout ressortissant d'un pays non européen admis dans un État de l'UE pour moins de trois mois. Tout dépassement de la durée du séjour autorisée donnera lieu à un signalement automatique aux autorités compétentes. Deux objectifs sont assignés à ce dispositif : d'une part, exercer un effet dissuasif sur les candidats à l'immigration irrégulière sous couvert d'un visa touristique. D'autre part, améliorer la connaissance concernant « les caractéristiques des dépassements (itinéraires, déclarations de prise en charge frauduleuses, pays d'origine et motifs du voyage), ainsi que [celle] des chiffres sur les flux migratoires et les personnes dépassant la durée de séjour autorisée, qui seront utiles à l'élaboration de la politique des visas " [17]. Déjà mentionnée, la privatisation des contrôles renforce ce maillage, puisque les compagnies aériennes et les agences de voyage qui collectent des informations individuelles sur les passagers les stockent dans les bases de données des systèmes de réservation (Passenger Name Record, PNR), ce qui permet leur partage au niveau international entre les entreprises intervenantes [18].

Avec la surveillance mise en place tout au long des routes empruntées par les migrants, on assiste à une remise en cause profonde du modèle traditionnel de la frontière qui, plus qu'une barrière, en fait un filtre entre les « touristes » et les « vagabonds », selon la distinction établie par Zygmunt Baumann [19].

\section{Les frontières internes}

Depuis que l'immigration est de plus en plus soumise aux contrôles administratifs, les frontières se sont déplacées des guérites des limites nationales aux guichets des centres-villes. Dès l'entre-deux-guerres, la situation des immigrés en France et leurs possibilités de se maintenir sur le territoire dépendaient peu de leurs capacités à traverser des délimitations interétatiques très perméables. Elles résultaient bien plus de leur pouvoir de négociation avec des fonctionnaires subalternes et des logiques bureaucratiques d'administrations sous-dimensionnées pour atteindre les objectifs qui leur étaient fixés [20]. En dépit de la montée en puissance de ces organismes de contrôle de l'immigration, cette situation n'a pas fondamentalement changé [21] : les faibles taux d'exécution des reconduites aux frontières et l'importance du retour des expulsés disent assez cette incapacité à refouler durablement au-delà des frontières extérieures. Ce sont bien des barrières internes qui constituent aujourd'hui les dispositifs-clés de séparation entre les nationaux et les étrangers - ou les personnes perçues comme telles. Les règlements européens qui, au nom de l'effacement des frontières internes de l'espace Schengen, ont permis de transformer les villes en vastes espaces de contrôle d'identité - par l'assimilation des gares internationales et de leurs périphéries à des zones frontières - sont l'incarnation juridique de ces fractures internes dont les effets sociaux sont ravageurs. Ainsi les frontières françaises, plus que de tracer un espace physique, traversent-elles le corps social, en raison notamment de segmentations raciales favorisées par les contrôles au faciès et la chasse aux sans-papiers [22]. La logique ancestrale de contrôle de 
la mobilité des populations les plus pauvres est donc loin d'avoir disparu et se rejoue chaque jour tant aux limes des villes qu'à ceux d'une UE très élargie.

De même qu'il y a déplacement des frontières pour des raisons environnementales ou climatiques, de même les contrôles migratoires opèrent-ils de véritables mutations des limites territoriales. Une frontière migratoire se définirait donc comme englobant la limite de souveraineté (c'est-à-dire la frontière politique, dessinée généralement de façon linéaire sur une carte), les zones tampons (pays limitrophes, camps d'étrangers, espace à proximité d'une frontière où des migrants "sans papiers " sont tolérés) et les dispositifs de contrôles situés dans les pays tiers. C'est le fonctionnement global de tous ces systèmes, autrement dit l'ensemble des dispositifs visant à contrôler les mouvements des migrants, qui fait sens.

La frontière migratoire est donc très étendue, et sur ces marges, elle évolue au gré des circonstances. Depuis une vingtaine d'années, celles des États « riches " se transforment sous l'influence des flux migratoires internationaux, face auxquels ils modifient leurs installations et créent de nouveaux dispositifs de surveillance. Les barrières migratoires ne sont plus la seule enveloppe externe des territoires, elles se matérialisent aujourd'hui par une série d'obstacles qui ponctuent le parcours des exilés à la recherche d'une vie meilleure ou d'une protection dans un autre État que le leur. En pratique, elles sont un redoutable frein à la liberté d'aller et venir.

\section{Notes}

[1] Sur l'invention de la dactyloscopie et des techniques anthropométriques d'identification des personnes, voir le site www.projetbertillon.com.

[2] About Ilsen, «De la libre circulation au contrôle permanent. Les autorités françaises face aux mobilités tsiganes transfrontalières, 1860-1930 », Culture \& Conflits, $n^{\circ} 76,2009$, p. 15-38.

[3] Michel Foucher, L'Obsession des frontières, Paris, Perrin, 2007.

[4] Ce programme qui s'appuie sur l'identification biométrique synthétisée dans un numéro et un dossier uniques conservés dans une gigantesque base de données est appelé IVFRT (Immigration, Visas, Foreigners'Registration and Tracking Project)

[5] Jacques Ancel, Géographie des frontières, Paris, Gallimard, 1938,209 p. ; Gottmann Jean, La Politique des États et leur géographie, Paris, Armand Colin, 1952, 228 p.

[6] Paul Guichonnet et Claude Raffestin, Géographie des frontières, Paris, PUF, 1974, 224 p.

[7] Olivier Clochard, «Le contrôle des flux migratoires aux frontières de l'Union européenne s'oriente vers une disposition de plus en plus réticulaire », Carnets de géographes, n 1, 2010.

[8] Habibou Bangré, « Le grillage de la discorde Le Botswana se protège du Zimbabwe », Afrik. com, 11 septembre 2003.

[9] Olivier Weber, « Ces murs qui divisent », Le Point, 10 janvier 2008.

[10] Migreurop, «Émigration illégale » : une notion à bannir, 2006, http:// www.migreurop.org/article922.html

[11] Thomas Hammarberg, « Les compagnies aériennes ne sont pas des services de l'immigration », 12 oct. 2010

[12] Cimade, Visa refusé. Enquête sur les pratiques consulaires en matière de délivrance des visas, 2010, 132 p.

[13] Malcolm Anderson, « Les frontières : un débat contemporain », Cultures \& Conflits, vol. 26-27, 1997

[14] Rappelons que la convention de Genève reconnaît aux demandeurs d'asile la possibilité de franchir les frontières sans document de voyage.

[15] Migreurop, « Derrière le mot camp », 16 nov. 2004

[16] Système d'information Schengen (SIS), Eurodac, Système d'information sur les visas (VIS).

[17] Commission européenne, Préparer les prochaines évolutions de la gestion des frontières dans l'Union européenne, $\mathrm{COM} / 2008 / 0069$ final.

[18] Iserte Morgane, « Enquête en "zone d'attente réservée" de l'aéroport de Paris-Charles-de-Gaulle : vers une gestion sécuritaire 
des « flux migratoires », Cultures \& Conflits 2008/3, n 71, 2008.

[19] Zygmunt Bauman, Le Coût humain de la mondialisation, Paris, Hachette Littératures, 1999.

[20] Mary Lewis, Les Frontières de la République. Immigration et limites de l'universalisme en France (1918-1940), Marseille, Agone, 2010.

[21] Alexis Spire, Accueillir ou reconduire. Enquête sur les guichets de l'immigration, Paris, Raisons d'agir, 2008.

[22] Didier Fassin (dir.), Les nouvelles frontières de la société française, Paris, La Découverte, 2010. 